\title{
Internações hospitalares por traumatismo cranioencefálico: uma análise do perfil epidemiológico no estado do Maranhão entre 2016 e 2020
}

\author{
Hospital admissions for traumatic brain injury: an analysis of the epidemiological profile in the
} state of Maranhão between 2016 and 2020

Ingresos hospitalarios por traumatismo craneoencefálico: un análisis del perfil epidemiológico en el estado de Maranhão entre 2016 y 2020

\author{
Matheus Machado de Asevêdo \\ ORCID: https://orcid.org/0000-0001-6116-2116 \\ Universidade Federal do Maranhão, Brasil \\ E-mail: matheus-machado97@hotmail.com \\ Sueli de Souza Costa \\ ORCID: https://orcid.org/0000-0003-4127-7324 \\ Universidade Federal do Maranhão, Brasil \\ E-mail: scsueli@gmail.com
}

\begin{abstract}
Resumo
Introdução: O traumatismo cranioencefálico (TCE) é caracterizado como importante causa de mortalidade e morbidade, deficiência mental e física, além de doença com grande impacto na qualidade de vida dos indivíduos que sofrem por esse trauma. Objetivos: Avaliar o perfil epidemiológico das internações hospitalares por traumatismo cranioencefálico no Estado do Maranhão entre os anos de 2016 e 2020. Metodologia: Trata-se de um estudo ecológico exploratório de base populacional que utilizou dados secundários sobre informações da população que é internada com TCE registradas no Sistema de Internações Hospitalares online do Sistema Único de Saúde (SIH/SUS), disponibilizadas no site do DataSUS. Resultados: A faixa etária de 20 a 39 anos de idade representou o maior número de casos de internações por TCE (42,39\%); quanto a prevalência por 100 mil habitantes, as maiores taxas foram na faixa etária daqueles com 80 anos ou mais, que apresentou um aumento de 32,19\% de 2016 a 2020; no perfil de lesão traumática, a maior prevalência corresponde aos TCE's não especificados (outros traumatismos intracranianos), sendo responsável por 56,49\% dos casos de internações; no que se refere à distribuição geográfica das internações hospitalares as maiores concentrações foram nas microrregiões de São Luís, seguida de Imperatriz. Conclusão: Os homens em idade laboral são os mais afetados no Maranhão, em concordância com outras pesquisas no país. Estudos sistematizados são necessários, a fim de se estabelecer políticas públicas de educação em saúde mais eficazes para reduzir as taxas de prevalência de TCE. Palavras-chave: Lesão Cerebral Traumática; Epidemiologia; Internação Hospitalar.
\end{abstract}

\begin{abstract}
Introduction: The traumatic brain injury (TBI) is characterized as an important cause of mortality and morbidity, mental and physical disability, in addition to a disease with a great impact on the quality of life of individuals who suffer from this trauma. Objectives: To evaluate the epidemiological profile of hospital admissions for traumatic brain injury in the State of Maranhão between 2016 and 2020. Methodology: This is an exploratory ecological population-based study that will use secondary data on information from the population that is hospitalized with TBI registered in the online Hospital Admission System of the Unified Health System (SIH/SUS), available on the website of DataSUS. Results: The age group from 20 to 39 years of age represented the largest number of cases of hospitalizations for TBI (42.39\%); as for the prevalence per 100,000 inhabitants, the highest rates were in the age group of those aged 80 years or more, which showed an increase of $32.19 \%$ from 2016 to 2020; in the traumatic injury profile, the highest prevalence corresponds to unspecified TBI's (other intracranial trauma), accounting for 56.49\% of hospital admissions; with regard to the geographic distribution of hospital admissions, the highest concentrations were in the microregions of São Luís, followed by Imperatriz. Conclusion: Men of working age are the most affected in Maranhão, in agreement with other surveys in the country. Systematized studies are needed in order to establish more effective public health education policies to reduce TBI prevalence rates.
\end{abstract}

Keywords: Traumatic Brain Injury; Epidemiology; Hospital internment.

\section{Resumen}

Introducción: El traumatismo craneoencefálico (TCE) se caracteriza por ser una importante causa de mortalidad y morbilidad, discapacidad mental y física, además de una enfermedad con un gran impacto en la calidad de vida de las 
personas que padecen este trauma. Objetivos: Evaluar el perfil epidemiológico de los ingresos hospitalarios por traumatismo craneoencefálico en el estado de Maranhão entre 2016 y 2020. Metodología: Se trata de un estudio poblacional exploratorio ecológico que utilizó datos secundarios sobre información de la población que se encuentra hospitalizada con TCE registrada en el sistema de ingresos hospitalarios en línea del Sistema Único de Salud (SIH / SUS), disponible en el sitio web de DataSUS. Resultados: El grupo de edad de 20 a 39 años representó el mayor número de casos de hospitalizaciones por TCE (42,39\%); en cuanto a la prevalencia por 100.000 habitantes, las mayores tasas se dieron en el grupo de edad de 80 años o más, que mostró un aumento del 32,19\% de 2016 a 2020; en el perfil de lesión traumática, la mayor prevalencia corresponde a TCE (otros traumatismos intracraneales) no especificados, representando el 56,49\% de los ingresos hospitalarios; En cuanto a la distribución geográfica de los ingresos hospitalarios, las mayores concentraciones se dieron en las microrregiones de São Luís, seguida de Imperatriz. Conclusión: Los hombres en edad de trabajar son los más afectados en Maranhão, de acuerdo con otras encuestas en el país. Se necesitan estudios sistematizados para establecer políticas de educación en salud pública más efectivas para reducir las tasas de prevalencia de TCE.

Palabras clave: Lesión cerebral traumática; Epidemiología; Internación hospitalaria.

\section{Introdução}

O traumatismo cranioencefálico (TCE) é conceituado como qualquer agressão gerada por forças externas que têm a capacidade de lesão anatômica ou comprometimento funcional de estruturas do crânio ou do encéfalo (Magalhães et al., 2017). Por apresentar taxas elevadas de morbimortalidade, é considerado um problema mundial de saúde pública e afeta em grande parte a população de faixa etária mais ativa (Nascimento et al., 2020).

A estimativa da incidência de TCE nos Estados Unidos varia em torno de 538 por 100 mil habitantes, a qual representa pelo menos 1,7 milhões de novos casos por ano desde 2003, custando ao governo, considerando-se somente os casos de TCE leves e moderados (75\% do total), cerca de 17 bilhões de dólares por ano (Magalhães et al., 2017).

Segundo Xenofonte e Marques (2021), no Brasil, os adultos jovens e os adolescentes são os indivíduos que compõem as principais vítimas de acidentes automobilísticos que resultam em TCE; no Nordeste do país há uma forte associação entre acidentes motociclísticos e TCE, sendo de especial interesse da saúde pública por conta do elevado número de internações diárias e procedimentos hospitalares por tal trauma.

Acidentes automobilísticos, quedas e as agressões físicas estão entre os principais fatores etiológicos de TCE, no entanto essas etiologias variam de acordo com o local de ocorrência (Ahmed et al., 2017). Dados brasileiros apontam as causas externas como uma das quatro razões mais frequentes de mortalidade no país e, se fossem excluídas as mortes por causas sem definição clara, ocupariam, então, o segundo ou terceiro lugares (Vale e Silva et al., 2018).

Os acidentes motociclísticos, como mecanismos geradores de trauma, têm relação íntima com indivíduos com menos de 45 anos de idade, ao passo que nos extremos de idade (população pediátrica e indivíduos com mais de 65 anos), as quedas estão mais associadas. O TCE apresenta impacto relevante na saúde por conta das perdas pessoais e socioeconômicas, que provocam marcantes sequelas neurodegenerativas, incapacitantes e irreversíveis (Rodrigues et al., 2018).

No Brasil mais de um milhão de pessoas vivem com sequelas neurológicas provocadas por TCE e que são de caráter irreversível, além disso mostra elevada e crescente incidência no mundo moderno particularmente nos países em desenvolvimento (Magalhães et al., 2017).

As pessoas que sobrevivem a um TCE podem ficar com sequelas permanentes incluindo déficits motores, sensoriais, cognitivos, de linguagem, emocionais e/ou comportamentais. Estudos indicam que cerca de 50\% a 75\% dos pacientes com TCE apresentam alterações cognitivas e comportamentais. Estas sequelas provocam um grande impacto para o indivíduo, sua família e sociedade (Arruda et al., 2015, p.56).

O TCE pode ser classificado de acordo com o nível de gravidade em leve, moderado e grave, através da Escala de Coma de Glasgow (ECG), a qual foi descrita pela primeira vez em 1974 por Teasdale e Jennett, e representa o parâmetro atualmente mais utilizado em âmbito mundial para avaliação do nível de consciência, pois tem, entre as suas principais vantagens, a 
reprodutibilidade de exames físicos fáceis de serem realizados (Morgado \& Rossi, 2011). As taxas de mortalidade no tipo grave variam de 30\% a 40\%, além de causar deficiências psicossociais e físicas em cerca de 60\% dos casos (Khellaf et al., 2019).

Através de pesquisas no Departamento de Informática do Sistema Único de Saúde (SUS) (DATASUS) os dados mostram que entre janeiro de 2008 e setembro de 2018, houve no Brasil a marca de 1.090.258 internações por traumatismos intracranianos, com predominância nas seguintes regiões: Sudeste, correspondendo a 42,5\% (463.396), e Nordeste do país, com 25,8\% (281.907) (Constâncio et al., 2018).

Os dados apresentados evidenciam a necessidade de uma compreensão a respeito da incidência desse tipo de trauma e do quanto ele deve ser foco de preocupação dos profissionais de saúde, sejam eles da atenção básica ou aqueles que têm atuação nos ambulatórios e hospitais, para que dessa forma exerçam coparticipação na redução desse importante agravo para a sociedade. Isso reflete-se na implementação de investimentos em políticas de prevenção de acidentes através de educação no trânsito, assim como educação em saúde (Filho et al., 2019).

Diante do exposto, é notável a relevância que os quadros de TCE apresentam no âmbito sócioeconômico, além das elevadas taxas de incidência, bem como de prevalência no Brasil, com destaque para a região Nordeste que apresenta um dos maiores números de internações diárias do país. No entanto, ainda há uma escassez de estudos que retratem os dados epidemiológicos sobre tal tipo de trauma. Logo, o objetivo desta pesquisa é traçar o perfil epidemiológico das internações hospitalares por traumatismo cranioencefálico no Estado do Maranhão entre os anos de 2016 e 2020; identificar a faixa etária e demais características da população estudada que mais interna na rede hospitalar devido a traumatismo cranioencefálico; analisar as taxas de prevalência das internações hospitalares por TCE de acordo com a faixa etária e o ano no Estado do Maranhão; além de comparar as microrregiões do Maranhão para constatar onde se concentram as maiores taxas de internações por TCE.

\section{Metodologia}

Trata-se de um estudo ecológico exploratório de base populacional, que consiste na comparação entre a ocorrência de uma condição relacionada à saúde e a exposição de interesse entre agregados de indivíduos com a finalidade de verificar a possível existência de associação entre elas (Brasil, 2003). Este tipo de estudo é realizado para comparar a "ocorrência da doença/condição relacionada à saúde e a exposição de interesse entre agregados de indivíduos (populações de países, regiões ou municípios, por exemplo) para verificar a possível existência de associação entre elas" (Lima-Costa \& Barreto, 2003). Foram utilizados dados secundários sobre informações da população que é internada com Traumatismo Cranioencefálico (TCE) registradas no Sistema de Internações Hospitalares online do Sistema Único de Saúde (SIH/SUS), disponibilizadas no site do DATASUS (Brasil, 2021). Foram consideradas as informações dos indivíduos que atendem aos seguintes critérios: ser residente do Maranhão e ter sido internado no Estado entre os anos de 2016-2020, devido a TCE com dados registrados no SIH/SUS com os seguintes códigos da Décima Revisão da Classificação Internacional de Doenças e Causas de Mortalidade (CID-10): (S06; S06.0 a S06.9) (OMS, 1994). Foram excluídos todos os casos que apresentaram dados incompletos para as análises propostas no presente estudo.

A taxa de prevalência das internações hospitalares foi mensurada por meio da coleta de dados sobre as pessoas internadas por TCE no Maranhão no período de janeiro de 2016 a dezembro de 2020, através do banco de dados online do SIH/SUS, ambos do Ministério da Saúde (Brasil, 2008). Com base nisso, a taxa de prevalência por 100 mil habitantes foi obtida através da fórmula matemática:

$$
\left(\frac{\text { população de determinada faixa etária internada por TCE }}{\text { população estimada da mesma faixa etária }}\right) \times 100000
$$


Da mesma forma que a taxa de prevalência, foi obtida a proporção das internações hospitalares na população que teve TCE em relação a todas as internações de acordo com a faixa etária. A partir disso, obteve-se a prevalência através da fórmula matemática:

$$
\left(\frac{\text { número de casos de internações por TCE }}{\text { total de internações }}\right) \times 100
$$

A análise da distribuição geográfica realizou-se através do software gratuito $Q g i s ®$, aplicando a estatística quantile. Os shapefiles (mapas) foram coletados no banco de dados do IBGE, e as proporções de pessoas internadas por TCE obtidas de cada microrregião do estado do Maranhão foram agregadas aos mapas.

Dessa forma, foram elaborados dois mapas, considerando o total de internações em números absolutos e a proporção de internações hospitalares por TCE, a fim de comparar se houve coincidência entre as microrregiões. Cada mapa foi dividido em quartis representados por diferentes cores, as mais claras (menores proporções) e as mais escuras (maiores proporções).

Além disso, foram elaboradas três tabelas utilizando os softwares Microsoft excel@ e Stata®. A primeira consta o número de casos de internações por TCE estratificados por faixa etária (menor que 20 anos; de 20 a 39 anos; de 40 a 59 anos; 60 a 79 anos e maiores que 80 anos), sexo e raça/cor. A segunda tabela contém a evolução da doença entre os anos 2016 e 2020 segundo as faixas etárias mencionadas, de acordo com a taxa de prevalência por 100 mil habitantes. A terceira tabela mostra as prevalências das internações de acordo com tipo de TCE, conforme a Classificação Internacional de Doenças (CID-10).

Por se tratar de pesquisa com dados secundários de domínio público, disponíveis em plataforma digital do DATASUS, este trabalho não necessitou ser submetido a um Comitê de Ética em Pesquisa, de acordo com a resolução 466/2012 do Conselho Nacional de Saúde, do Ministério da Saúde.

\section{Resultados}

O estudo revelou um total de 18.499 casos de TCE que necessitaram de internação no Estado do Maranhão no período de 2016-2020, evidenciado na Tabela 1. A faixa etária de 20 a 39 anos de idade representou o maior número de casos de internações por TCE sendo responsável por 7.842 casos (42,39\%). Já a faixa etária daqueles menores que 20 anos foi a segunda mais prevalente em números absolutos apresentando 4.032 casos $(21,80 \%)$ seguida daqueles entre 40 e 59 anos com 3.772 internações (20,39\%) e 60 a 79 anos com 2.167 casos (11,71\%). A faixa etária daqueles com 80 anos ou mais foi a que apresentou o menor número de casos com 686 (3,71\%). Em relação ao sexo, os homens representaram 76,81\% das internações por TCE com 14.209 casos. Quanto a raça/cor mais prevalente, tem-se os pardos com 5.285 (28,57\%), o percentual de ignorados foi de $54,49 \%$. 
Tabela 1. Prevalência das internações hospitalares por Trauma Cranioencefálico de acordo com a faixa etária, sexo e raça/cor no Estado do Maranhão no período de 2016 a 2020.

\begin{tabular}{lcc} 
Características & Internações hospitalares por TCE \\
\hline Faixa etária & $(\mathbf{n = 1 8 . 4 9 9 )}$ & $(\mathbf{\%})$ \\
\hline Menores que 20 anos & 4.032 & 21,80 \\
de 20 a 39 anos & 7.842 & 42,39 \\
de 40 a 59 anos & 3.772 & 20,39 \\
de 60 a 79 anos & 2.167 & 11,71 \\
Maior ou igual a 80 anos & 686 & 3,71 \\
\hline Sexo & & \\
\hline Masculino & 14.209 & 76,81 \\
Feminino & 4.290 & 23,19 \\
\hline Raça/Cor & & 3,23 \\
\hline Branco(a) & 598 & 1,59 \\
Preto(a) & 294 & 28,57 \\
Pardo(a) & 5.285 & 11,91 \\
Amarelo(a) & 2.203 & 0,21 \\
Indígena & 38 & 54,49 \\
Ignorado(a) & 10.081 & $\mathbf{1 0 0}$ \\
\hline Total & $\mathbf{1 8 . 4 9 9}$ & \\
& & \\
\hline & & \\
\hline
\end{tabular}

Fonte: Elaborado pelo autor, com dados oriundos do Ministério da Saúde - SIH/SUS (2021).

Com relação às taxas de prevalência por 100 mil habitantes das internações por TCE de acordo com a faixa etária e o ano, é evidenciado na Tabela 2 a evolução dos casos no período de 2016 a 2020 . Ao compararmos o período de 2016 e de 2020 , foi constatada uma redução de 2,33\% dos casos na faixa etária de menores que 20 anos. Naqueles pertencentes ao grupo dos adultos entre 20 e 39 anos, houve uma redução de 1,92\%. No entanto, na faixa etária de 40 a 59 anos, foi observado um aumento de $22,54 \%$. Nas faixas etárias pertencentes ao grupo dos idosos, ou seja, maiores que 60 anos, foi evidenciado que naqueles entre 60 e 79 anos de idade ocorreu um aumento de 10,89\%. As maiores taxas foram na faixa etária daqueles com 80 anos ou mais, que apresentou um aumento de 32,19\%, chegando a atingir 179,16 casos/100 mil hab. no ano de 2018. Por sua vez, a menor taxa foi observada na faixa etária de menores que 20 anos com 30,05 casos/100 mil hab. no ano de 2020. Outro ponto a ser observado é que o ano de 2019 apresentou as maiores taxas em todas as faixas etárias, com exceção daqueles com 80 anos ou mais. 
Tabela 2. Prevalência por 100 mil habitantes das internações hospitalares por Traumatismo Cranioencefálico (TCE) de acordo com a faixa etária e o ano no Estado do Maranhão.

\begin{tabular}{lccccc} 
Faixa etária & \multicolumn{5}{c}{ Ano } \\
\hline Menores que 20 anos & $\mathbf{2 0 1 6}$ & $\mathbf{2 0 1 7}$ & $\mathbf{2 0 1 8}$ & $\mathbf{2 0 1 9}$ & $\mathbf{2 0 2 0}$ \\
de 20 a 39 anos & 30,77 & 31,84 & 31,37 & 33,40 & 30,05 \\
de 40 a 59 anos & 66,93 & 63,97 & 64,06 & 68,09 & 65,64 \\
de 60 a 79 anos & 47,32 & 49,97 & 57,91 & 58,65 & 57,99 \\
Maior ou igual a 80 anos & 62,51 & 66,72 & 80,06 & 83,19 & 69,32 \\
\hline
\end{tabular}

Fonte: Elaborado pelo autor, com dados oriundos do Ministério da Saúde - SIH/SUS (2021).

Por sua vez, a Tabela 3 evidencia o número de internações hospitalares por TCE de acordo com os tipos de lesões presentes no CID-10. Diante disso, foi possível observar que a maior prevalência corresponde aos TCE's não especificados (outros traumatismos intracranianos - S06.8), sendo responsável por 56,49\% dos casos de internações. As outras causas de internações mais prevalentes relacionadas ao TCE foram o edema cerebral traumático (S06.2) com 10,76\% dos casos, Hemorragia subdural devida a traumatismo (S06.5) com 6,08\%, Concussão cerebral (S06.0) com 4,64\% e o Traumatismo cerebral focal (S06.3) com 4,49\%.

Tabela 3. Prevalência das internações hospitalares por Traumatismo Cranioencefálico (TCE) conforme a $10^{a}$ edição do Código Internacional de Doenças (CID-10) no Maranhão no período de 2016 a 2020.

\begin{tabular}{lcc} 
Classificação de acordo com o CID-10 & Frequência dos TCE's \\
\hline Traumatismo intracraniano (S06) & $(\mathbf{n = 1 8 . 4 9 9 )}$ & (\%) \\
Concussão cerebral (S06.0) & 26 & 0,14 \\
Edema cerebral traumático (S06.1) & 859 & 4,64 \\
Traumatismo cerebral difuso (S06.2) & 1.990 & 10,76 \\
Traumatismo cerebral focal (S06.3) & 584 & 3,16 \\
Hemorragia epidural (S06.4) & 831 & 4,49 \\
Hemorragia subdural devida a traumatismo (S06.5) & 682 & 3,69 \\
Hemorragia subaracnóide devida a traumatismo (S06.6) & 1.125 & 6,08 \\
Traumatismo intracraniano com coma prolongado (S06.7) & 172 & 0,93 \\
Outros traumatismos intracranianos (S06.8) & 9 & 0,05 \\
Traumatismo intracraniano, não especificado (S06.9) & 10.451 & 56,49 \\
\hline Total & 1.770 & 9,57 \\
\hline
\end{tabular}

Fonte: Elaborado pelo autor, com dados oriundos do Ministério da Saúde - SIH/SUS (2021).

No que se refere à distribuição geográfica das internações hospitalares por TCE em números absolutos e proporções em relação ao total de internações no Estado do Maranhão no período de 2016 a 2020, observou-se que as maiores concentrações foram nas microrregiões de São Luís (2.607 casos), seguida de Imperatriz (2.503 casos), Pindaré (2.142 casos), Baixada Maranhense (1.358 casos) e Alto Mearim e Grajaú (1.204 casos) (Figura 1-1A). A Figura 1B evidencia as proporções de internações por TCE. Houve uma mudança na distribuição dos casos ao comparar com os números absolutos, sendo que as maiores proporções observadas estão nas microrregiões do Litoral Ocidental Maranhense (1,48\%), Imperatriz (1,33\%), Codó 
(1,20\%), Gurupi Maranhense (1,15\%) e Presidente Dutra (1,08\%). Foi observado uma coincidência apenas da microrregião de Imperatriz que ocupou as cinco primeiras colocações no ranking de proporções de internações e números absolutos por TCE.

Figura 1. Internações Hospitalares por TCE no Estado do Maranhão no período de 2016 a 2020. 1A: internações hospitalares por TCE em números absolutos. 1B: Proporção de internações hospitalares por TCE em relação ao total de internações.

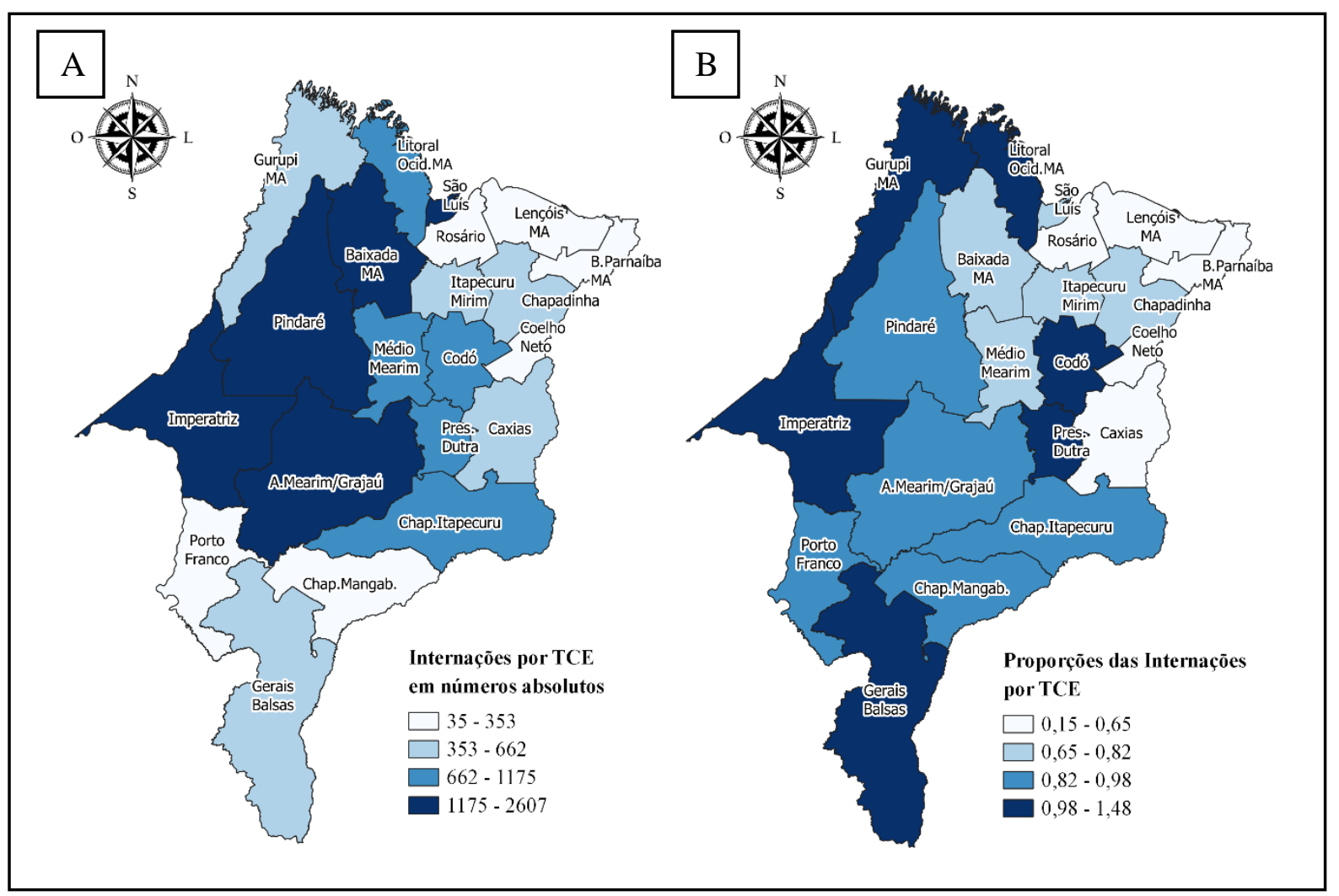

Fonte: Elaborado pelo autor, com dados oriundos do Ministério da Saúde - SIH/SUS (2021).

\section{Discussão}

O TCE é uma causa de internação frequente em jovens, com uma prevalência maior no sexo masculino (Ahmed et al., 2017). No presente estudo nota-se que de um total absoluto de 18.499 internações hospitalares por TCE no período proposto, mais de $70 \%$ eram do sexo masculino, contra 23,19\% correspondendo ao sexo feminino, com um predomínio de TCE em homens de 3,31 vezes maior do que em mulheres. Tal análise reforça estudos anteriores que evidenciaram o sexo masculino como o mais afetado, como o de Santos (2020), de âmbito nacional. Cabe ainda destacar que análises referentes especificamente à região Nordeste também apresentaram maior prevalência em homens, em uma pesquisa realizada por Xenofonte e Marques (2021), no período entre 2009 e 2019.

Os resultados acima contribuem para reiterar o fato de os homens estarem mais predispostos à principal causa de TCE no Brasil, que corresponde aos acidentes automobilísticos, com destaque para os motociclísticos, os quais apresentam altas taxas de morbidade e mortalidade. Além disso, características culturais que colocam o homem em contextos de maior vulnerabilidade, como situações violentas aliadas ao uso abusivo de álcool, corroboram com os altos índices de internação nessa população (Xenofonte \& Marques, 2021).

Com relação à faixa etária, o presente estudo destaca que 42,39\% foram correspondentes aos indivíduos de 20 a 39 anos, ou seja, a grande maioria dos casos se concentra na parcela produtiva da sociedade. Em contrapartida, notou-se que aqueles 
com idade maior ou igual a 80 anos apresentaram as menores taxas, totalizando 3,71\% do total. O contrário foi observado em um estudo europeu, no qual evidencia-se taxas mais elevadas de internações na faixa etária acima de 80 anos (Madjan, et al., 2016). Importante pontuar que uma das grandes causas de TCE nos idosos são as quedas, provocadas principalmente por conta da fraqueza muscular e óssea que essas pessoas sofrem com o passar dos anos.

Por atingir principalmente a população economicamente ativa, configura-se como um desafio aos gestores de políticas públicas, além de ser a terceira causa de morte no país (Nascimento et al., 2020). Vale ressaltar ainda a faixa etária dos menores de 20 anos, na qual se encontra a população pediátrica, que apresentou o segundo lugar com 4.032 casos. É importante pontuar que o TCE é responsável por $75 \%$ das internações por causas externas em crianças, e entre as causas as quedas correspondem a $35 \%$ dos casos (Furlan et al., 2019).

No tocante à raça constatou-se que os pardos foram os mais prevalentes, com 28,57\%, excluindo os ignorados com 54,49\%. Estes resultados assemelham-se a outros estudos regionais como o de Sousa e Silva et al. (2019), que também chegou a esta conclusão no estado da Bahia entre 2008 a 2017. A raça amarela ficou em segundo lugar em número de internações, totalizando $11,91 \%$ com 2.203 casos. A raça indígena, por sua vez, quando comparada com todas as outras raças apresentou taxas inexpressivas, com 38 casos do total, perfazendo $0,21 \%$.

Sabe-se que há um significativo impacto tanto de caráter social como econômico associado aos casos de TCE, e isso é observado mais substancialmente em países pobres e em desenvolvimento, os quais apresentam altas taxas de prevalência e incidência que aumentam com os anos, porém a escassez de estudos epidemiológicos limita um conhecimento mais a fundo sobre a real situação brasileira (Magalhães et al., 2017). Foram encontrados poucos artigos de âmbito nacional, a grande maioria baseia-se em dados regionais e nenhum deles abordou especificamente a prevalência por 100 mil habitantes por faixa etária durante os anos analisados, o que reforça a necessidade de mais estudos de caráter epidemiológico no país.

Vale frisar que a população idosa acima de 80 anos apresentou as maiores taxas de prevalência por 100mil habitantes em todos os anos, com um aumento de 32,19\% de 2016 para 2020. Apesar da escassez de pesquisas que abordem esse tópico específico, sabe-se que os idosos apresentam uma média de permanência hospitalar maior que as outras, uma vez que a senilidade acaba por exigir maiores assistências à saúde. Tal fato poderia explicar as altas taxas de prevalência nessa população e essa análise pode ter outros resultados em regiões com menor ou maior quantidade de idosos (Santos, 2020).

É importante destacar novamente que são poucos os estudos epidemiológicos sobre TCE no Brasil, o que limita análises comparativas deste importante problema de saúde pública com parâmetros específicos. No que diz respeito ao perfil de lesões traumáticas por TCE com base no CID-10, a maior prevalência foi encontrada no descritor S06.8 (outros traumatismos intracranianos) com 10.451 casos, seguido por edema cerebral traumático (S06.1) com 1.990 casos do total.

Foram encontradas apenas duas pesquisas brasileiras que abordam os tipos de lesão encefálica nas populações estudadas. No primeiro, realizado com dados de São Paulo no ano de 1997 a classificação em vigor era a CID-9, porém ainda foi possível fazer algumas correlações com o presente estudo. Naquele se chegou à conclusão de que 49,3\% dos internados tiveram diagnóstico com descritor 854 , que engloba tanto a lesão traumática craniana de outra natureza como os não especificados, ou seja, equivalente ao S06.8 do atual CID-10, que foi o mais prevalente do estudo atual. Tal resultado revela tanto uma imprecisão no diagnóstico da época quanto a importância na modificação no Código Internacional de Doenças para se ter números mais fidedignos com relação a diagnósticos específicos (Koizumi et al., 2000). O outro estudo, mais atual, realizado em um hospital de referência de Teresina no ano de 2015 apesar de abordar tipos de lesões encefálicas decorrentes de TCE, não utilizou os códigos da CID para nortear seus resultados, o que dificultou análises comparativas mais precisas (Santos et al., 2016).

Quanto à distribuição geográfica das internações no Maranhão, como ressaltado nos resultados, as maiores taxas foram em São Luís (2.607 casos), seguida de Imperatriz (2.503 casos), Pindaré (2.142 casos), Baixada Maranhense (1.358 casos) e Alto 
Mearim e Grajaú (1.204 casos). Muito provavelmente São Luís e Imperatriz ocupam os primeiros lugares por serem as cidades mais populosas do Estado, com a primeira totalizando mais de 1 milhão de habitantes e a segunda com mais de 200 mil habitantes (IBGE, 2021). Já no que diz respeito às proporções em relação ao total de internações houve uma mudança nos padrões, com os maiores índices presentes nas microrregiões do Litoral Ocidental Maranhense (1,48\%), Imperatriz (1,33\%), Codó $(1,20 \%)$, Gurupi Maranhense $(1,15 \%)$ e Presidente Dutra $(1,08 \%)$, com a necessidade de mais pesquisas de caráter epidemiológico para estabelecer relações comparativas e as causas dessa mudança.

\section{Conclusão}

O TCE é um grave problema de saúde pública, uma vez que atinge todas as faixas etárias e tem a capacidade de gerar consequências sociais, cognitivas e econômicas de caráter irreversível. Observou-se que os homens em idade laboral são os mais afetados no Maranhão, em concordância com outras pesquisas no país, o que mostra uma relação intrínseca dessa parcela populacional com atitudes imprudentes no trânsito, uma vez que é a principal causa de traumatismo craniano. Com relação às altas taxas de prevalência na população idosa, conclui-se que outras morbidades decorrentes do processo de senilidade favorecem um pior prognóstico nesses pacientes, aumentando o tempo de permanência hospitalar dos mesmos.

As cidades mais populosas do Estado apresentaram as maiores taxas de prevalência, o que reforça a necessidade de fortalecer as práticas de educação no trânsito, além de maior rigor quanto às leis que regem motoristas e pedestres com o intuito de minimizar os riscos de acidentes.

Assim, o presente estudo tem a finalidade de servir como incentivo a novas pesquisas, pois é notório a carência de estudos sistematizados de caráter nacional e regional para se ter um conhecimento mais amplo a respeito da situação epidemiológica de cada estado e do país por inteiro. Dessa forma, será possível estabelecer, com mais eficácia, políticas públicas de educação em saúde que reduzam as altas taxas de prevalência de TCE na sociedade, assim como os gastos com internação, poupando a maior parte das pessoas das graves consequências desta enfermidade.

Devido à importância da temática retratada, sugere-se que novas pesquisas sobre o perfil epidemiológico das internações por TCE no Maranhão e nos outros estados da federação, de caráter prospectivo, sejam desenvolvidas para favorecer a expansão do conhecimento técnico e científico, com a finalidade de esclarecer os pormenores sociais e econômicos que envolvem tal patologia, o que viabilizará medidas de controle mais efetivas.

\section{Referências}

Ahmed, S., Venigalla, H., Mekala, H. M., Dar, S., Hassan, M., \& Ayub, S. (2017). Traumatic Brain Injury and Neuropsychiatric Complications. Indian J Psychol Med, 39(2), 114-121. https://doi.org/10.4103/0253-7176.203129

Arruda, B. P., Akamatsu, P. Y. F., Xavier, A. P., Costa, R. C. V., Alonso, G. S. d. O., \& Madaleno, I. M. P. (2015). Traumatismo crânio encefálico e suas implicações cognitivas e na qualidade de vida. Acta Fisiátrica, 22(2), 55-59. https://doi.org/10.5935/0104-7795.20150012

Brasil (2008). Ministério da Saúde. Indicadores Básicos Para a Saúde No Brasil: Conceitos e Aplicações. 202-203. 2008

Brasil (2003). Ministério da Saúde. Secretaria de Vigilância em Saúde. Epidemiologia e serviços de saúde. Brasília: Ministério da Saúde, 12 (4). https://bvsms.saude.gov.br/bvs/periodicos/rev_epi_vol12_n4.pdf.

Brasil. Ministério da Saúde. Sistema de informações hospitalares do SIH/SUS. Brasília: Ministério da Saúde; 2021. http://datasus.saude.gov.br/informacoesde-saude/tabnet/epidemiologicas-e-morbidade.

Constâncio, J. F., Nery, A. A., Mota, E. C. H., Santos, C. A. d., Cardoso, M. C., \& Constâncio, T. O. d. S. (2018). Perfil clínico-epidemiológico de indivíduos com histórico de traumatismo cranioencefálico. Rev. baiana enferm, 32, Artigo e28235. https://doi.org/10.18471/rbe.v32.28235

Filho, R. F. d. S., Gonçalves, K. G., Araujo, J. A. M. d., Matos, T. A., Silva, H. K. S., \& Menezes, R. S. P. (2019). Perfil clínico-epidemiológico dos traumatismos cranioencefálicos atendidos em um hospital de referência do interior do estado do Ceará. Nursing (São Paulo), 22(253), 29092913. https://doi.org/10.36489/nursing.2019v22i253p2909-2913 
Furlan, F. L. S., Leme, M. A., Rodrigues, N. G. A., Longo, S. T., Azevedo, G., Pascolat, G., \& Cruz, A. S. d. (2019). Traumatismo cranioencefálico pediátrico no pronto atendimento: estudo dos critérios para realização de tomografia. Rev. Méd. Paraná, 77(2), 2735. https://pesquisa.bvsalud.org/portal/resource/pt/biblio-1283716

Instituto Brasileiro de Geografia e Estatística - IBGE. Cidades e Estados. Disponível em: https://www.ibge.gov.br/cidades-e-estados/ma/.html.

Khellaf, A., Khan, D. Z., \& Helmy, A. (2019). Recent advances in traumatic brain injury. Journal of Neurology, 266(11), 28782889. https://doi.org/10.1007/s00415-019-09541-4

Koizumi, M. S., Lebrão, M. L., Mello-Jorge, M. H. P. D., \& Primerano, V. (2000). Morbimortalidade por traumatismo crânio-encefálico no município de São Paulo, 1997. Arquivos de Neuro-Psiquiatria, 58(1), 81-89. https://doi.org/10.1590/s0004-282x2000000100013

Lima-Costa, M.F. \& Barreto, S.M. (2003). Tipos de Estudos epidemiológicos: conceitos básicos e aplicações na área do envelhecimento. Epidemiologia e Serviços de Saúde, 12(4), 189 - 201. http://scielo.iec.gov.br/pdf/ess/v12n4/v12n4a03.pdf

Magalhães, A., Cruz De Souza, L., Faleiro, R., Teixeira, A., \& Miranda, A. (2017). Epidemiologia do traumatismo cranioencefálico no Brasil. Revista Brasileira De Neurologia, 53(2). https://Revistas.Ufrj.Br/Index.Php/Rbn/Article/View/12305

Majdan, M., Plancikova, D., Brazinova, A., Rusnak, M., Nieboer, D., Feigin, V., \& Maas, A. (2016). Epidemiology of traumatic brain injuries in Europe: a cross-sectional analysis. The Lancet Public Health, 1(2), Artigo e76-e83. https://doi.org/10.1016/s2468-2667(16)30017-2

Morgado, F. L., \& Rossi, L. A. (2011). Correlação entre a escala de coma de Glasgow e os achados de imagem de tomografia computadorizada em pacientes vítimas de traumatismo cranioencefálico. Radiologia Brasileira, 44(1), 35-41. https://doi.org/10.1590/s0100-39842011000100010

Nascimento, S., Braga, G., Queiroz, A., Laureto, J., Campos, A., Macedo, J., \& Silva, P. (2020). Perfil epidemiológico de pacientes adultos com traumatismo cranioencefálico grave na rede SUS do Distrito Federal: um estudo retrospectivo. Revista Brasileira de Neurologia, 56(4). https://revistas.ufrj.br/index.php/rbn/article/view/40224

Organização Mundial da Saúde. Classificação estatística internacional de doenças e problemas relacionados à saúde: $10^{\mathrm{a}}$ revisão, São Paulo: centro colaborador da OMS para a classificação de doenças em português/Edusp; 1994. V. 2. Manual de instrução.

Santos, A. M. R. d., Sousa, M. E. d. C., Lima, L. O., Ribeiro, N. d. S., Madeira, M. Z. d. A., \& Oliveira, A. D. d. S. (2016). Traumatismo cranioencefálico no Brasil: análise epidemiológica. Rev. enferm. UFPE on line, 10(11), 3960-3968. https://periodicos.ufpe.br/revistas/revistaenfermagem/article/view/11478

Santos, J. d. C. (2020). Traumatismo cranioencefálico no brasil: análise epidemiológica. Revista Científica da Escola Estadual de Saúde Pública de Goiás Cândido Santiago, 6(3), Artigo e6000014. https://www.revista.esap.go.gov.br/index.php/resap/article/view/249

Souza e Silva, D. D. S., Oliveira, M. T. S., Maia, M. D. S., Lago, M. S., Pinheiro, E. N. d. S., Botelho, P. M., Lobo, J. O., De Oliveira, D. F., Picanço, C. M., \& Das Merces, M. C. (2019). Morbimortalidade hospitalar por traumatismo cranioencefálico na Bahia entre 2008 a 2017. Enfermagem Brasil, 18(5), 665. https://doi.org/10.33233/eb.v18i5.3075

Vale E Silva, L., Nogueira, T., Cunha, R., Monteiro, L., Monteiro, L., Mascarenhas, M., Oliveira Filho, O., \& Campelo, V. (2018). Análise das características de indivíduos com sequelas de traumatismo cranioencefálico (tce) em um centro de referência em reabilitação (características de tce). Revista Brasileira De Neurologia, 54(2). https://revistas.ufrj.br/index.php/rbn/article/view/19111

Rodrigues, M. d. S., Fernandes e Santana, L., Graça e Silva, E. P., \& Gomes, O. V. (2018). Epidemiologia de traumatismo craniencefálico em um hospital. Rev Soc Bras Clin Med, 16(1), 21-4. https://docs.bvsalud.org/biblioref/2018/06/884987/dezesseis1_vinteum.pdf

Xenofonte, M., \& Marques, C. (2021). Perfil epidemiológico do traumatismo cranioencefálico no Nordeste do Brasil. Revista Brasileira de Neurologia, 57(1). https://revistas.ufrj.br/index.php/rbn/article/view/43086 